\title{
Adjuvant chemotherapy for rectal cancer with complete pathological response (pCR) may not be necessary: a pooled analysis of 5491 patients
}

\author{
Xiang Hü ${ }^{1,2}$, Ya-Qi Li $i^{1,2}$, Xiao-ji Ma ${ }^{1,2}$, Long Zhang ${ }^{1,2}$, San-Jun Cai ${ }^{1,2}$ and Jun-Jie Peng ${ }^{1,2^{*}}$
}

\begin{abstract}
Background: It is recommended postoperative adjuvant chemotherapy for all rectal cancers undergoing neochemoradiotherapy regardless of the final yield pathology. However, the role of adjuvant chemotherapy in pathological complete response ( $\mathrm{pCR}$ ) remains controversial. We aimed to identify the necessarily of adjuvant chemotherapy in pCR.

Methods: Consecutive patients with pCR in Fudan University Shanghai Cancer Center (FUSCC) were enrolled. Meanwhile, a pooled analysis of individual patient with pCR was performed from PubMed and Embase databases for validation.

Results: A total of 171 patients form FUSCC were identified to achieve pCR with up to almost 10 years follow-up. Among them, those receiving adjuvant chemotherapy had no survival benefits compared to those without adjuvant chemotherapy (log-rank test $=0.17, \mathrm{P}=0.676$ ). The $5 y$-DFS rates for patients in chemo group and no-chemo group was 87.5 and $88.8 \%$, respectively, showing no significant difference $(p=0.854)$. No matter chemotherapy regimens, $T$ stage, EMVI and CRM status varied, the results remained consistent. Meantime, the COX model did not demonstrate adjuvant chemotherapy as the independent risk factor for OS and DFS. Additionally, among 18 systemic recurrences in all, the rate of relapse surged rapidly on the 12 months and rose up to peak in the 36th months. In order to validate these results, nine controlled trials involving 5491 patients with pCR were included in this pooled-analysis. For both 5-year overall survival and disease-free survival, the pooling data did not produce a statistically significant effect in cases of adjuvant chemotherapy performed ( $R R=0.79$ and $R R=0.95$, respectively, all $p>0.05)$.
\end{abstract}

Conclusion: This study suggested that rectal cancer patients with $\mathrm{PCR}$ did not benefit from adjuvant chemotherapy and we recommended that achievement of pCR require more prolonged close follow care in case of distant metastasis.

Keywords: Rectal cancer, Complete response, Adjuvant chemotherapy, Neo-adjuvant chemoradiotherapy, Survival

\footnotetext{
*Correspondence: pengjj67@hotmail.com

${ }^{1}$ Department of Colorectal Surgery, Fudan University Shanghai Cancer

Center, 270 Dong'an Road, Shanghai 20032, China

Full list of author information is available at the end of the article
} 


\section{Background}

Neo-adjuvant chemoradiotherapy (CRT) followed by radical surgery is the recommended optimal treatment for locally advanced rectal cancer [1], as the preoperative chemoradiotherapy could reduce recurrence and improve survival [2]. Similarly, post-operative adjuvant chemotherapy has an established role in patients with locally advanced rectal cancer for reducing the risk of recurrence and mortality up to $20-30 \%$ [3]. Then, is adjuvant chemotherapy necessary to the patients with complete response (pCR)? As once pCR has achieved, it will be followed with very low local recurrence, distant metastasis and improved long-term survival [4]. According to the present guidelines, adjuvant chemotherapy is offered as a standard treatment for all patients receiving CRT and the radical surgery, regardless of the postoperative pathological results. The NCCN recommended postoperative adjuvant chemotherapy for all patients undergoing preoperative chemoradiotherapy regardless of the final yield pathology, even pCR [5], while, the ESMO guidelines stated that "similar to the situation in colon cancer Stages III (and "high-risk" Stage II), adjuvant chemotherapy can be provided, even if the scientific support for sufficient effect is less" [6]. However, the various expert panels regarded definition of high risk differed slightly, generally including T4 tumor, poor differentiation, inadequate lymph node retrieval, the circumferential resection margin(CRM), extramural venous invasion (EMVI). Hence, no consensus was arrived on the basis of pCR in determining the necessity for adjuvant chemotherapy. Despite the widespread use of chemotherapy, solid evidence to support the benefits of adjuvant chemotherapy after pCR and radical excision is lacking [7]. What is more, several authors have demonstrated that $\mathrm{pCR}$ patients cannot benefit from the adjuvant chemotherapy after CRT and the curative resection [8-10], however their studies showed characteristic of small size sample and selection bias. With these heterogeneous results and potential harmful effects about chemotherapy, we determined to assess the value of adjuvant chemotherapy after achievement of $\mathrm{pCR}$ following preoperative radiology and surgery in rectal cancer patients.

\section{Materials and methods Data source}

Since 2006, all patients undergoing surgery for colorectal cancer at the Department of colorectal Surgery, Fudan University Shanghai Cancer Center (FUSCC), Shanghai, China, are scheduled for periodic follow-up at our cancer center. All patient data are prospectively entered in the FUSCC database, including age, race, tumor location, year of diagnosis, primary tumor size, histological grade, number of lymph nodes examined, type of radiation, marital status, preoperative multimodal treatment, details of the surgical procedure, occurrence of complications, postoperative histopathology, application of adjuvant therapy, and follow-up (date of last visit, tumor recurrence, date of tumor-related or unrelated death, overall and disease-free survival). The complete response was defined as ypT0NOM0 (absence of invasive cancer and in situ cancer in the rectum, reginal nodes and no imaging metastasis). The clinical staging prior operation was based on imaging studies including high resolution rectal magnetic resonance (MRI) and abdominopelvic contrast-enhanced computed tomography (CT) scan. Neo-adjuvant chemoradiotherapy regimen included a total of 50 Gy radiation (2 Gy per day for 25 fractions over 5 weeks) with concurrent chemotherapy of CapeOX or single-agent Capecitabine regimen. All patients from FUSCC dataset have provided written informed consent. The research protocol was reviewed and approved by the institutional review board of the FUSCC. Once the pCR was achieved, adjuvant chemotherapy or not will be based on the will of patients.

\section{Search strategy for systematic analysis}

A literature search was carried out in the electronic databases including PubMed, Web of Science, EMBASE and the Cochrane Central Register of Controlled Trials on February 1st, 2019. We employed the key words "rectal cancer, rectal carcinoma, neoplasms" and "chemotherapy, adjuvant" and "chemoradiotherapy, chemoradiotherapy, chemoradiation, radiotherapy" and "complete response, pCR, ypCR" with limits of "Clinical Trial" and "Humans". The related article references were also enriched the search. All available randomized controlled trials (RCTs) and comparative cohort studies evaluating the efficacy of adjuvant chemotherapy after pCR with at least one quantitative outcome were enrolled. The primary outcome was 3 or 5 -year OS, and the 3 or 5 -year DFS. Two authors independently extracted the below data: authors, publication year, study design, age, adjuvant chemo (schedules), median follow-up, OS, and DFS. Any disagreement was resolved by a senior author. When several studies by the same database reported the same outcomes at similar follow-up periods, we included in our analysis either the better quality or the most informative publication.

\section{Inclusion and exclusion criterion}

All clinical controlled trials, evaluating the value of adjuvant chemotherapy after achievement of pCR following neo-adjuvant chemoradiotherapy and surgery in rectal cancer patients, were selected for eligible. Trials were enrolled without limitation to nations, year, or language. We excluded studies in which the outcomes of interest were not reported, or it was impossible to 
calculate these from the published results. No control group, of course, was within our exclusion criteria.

The quality of included randomized trials were assessed as three categories from A (high quality) to C (low quality). These quality criteria included the randomization procedure, the use of intention-to-treat analysis, the report of dropout rates, allocation concealment and the extent to which valid outcomes were described. But this analysis included all case-control studies, which quality were not high.

\section{Statistical analysis}

Statistical evaluation was performed using IBM SPSS statistics Version 22 (SPSS Inc; IBM Corporation Software Group, Somers, NY). The Chi square test or Fisher exact test was utilized for exploratory comparisons of patient groups. All statistical tests were performed 2 -sided, and $\mathrm{p}$ values less than 0.05 were considered to be statistically significant. Observed (unadjusted overall) survival was estimated with the Kaplan-Meier method, and the log-rank (Mantel-Cox) test was used to compare independent subgroups. Overall survival and disease-free survival were used as the primary outcome parameter. Overall survival may represent disease-specific survival regarding the initial malignant disease and considers only tumor related deaths as events in this study [11]. Cox proportional hazard models were used to investigate the effect on survival of multivariable relationships among covariates including the age at diagnosis, gender, stage at diagnosis, EMVI, CRM and treatment. Stage, or any known clinical characteristics supposed to affect the prognosis were the stratified variable. Hazard ratios (HRs) and 95\% confidence intervals (CIs) for multivariate analyses were computed using the Cox proportional hazards regression models.

This systematic comparison was carried out in line with Cochrane Collaboration recommendations of meta-analyses guidelines [12]. For categorical variables, the relative risk (RR) as the summary statistics was employed, demonstrating the adverse ratio in the study group (chemo group) relative to control group (no-chemo group). A relative risk of less than one was the favor of the study group, and the point estimate of the relative risk was taken of statistical significance at the $\mathrm{p}=0.05$ level, if the $95 \%$ confidence interval did not include the value 1 . A fixed effects model was used on the presumption that variation in the individual trial results occurred about a true mean. Conversely, the randomized model was adopted. All statistical analyses were carried out with Stata 10 (StataCorp, College Station, Tex).

\section{Results}

Patient characteristics and tumor presentation in FUSCC

From the 950 patients with neo-adjuvant chemoradiotherapy and curative resection in the FUSCC colorectal cancer dataset from 2006 to 2016, we focused the rectal cancer patients with pCR with up to 10-year follow-up (11-138 months). There was a total of 171 patients with under peritoneal reflection who achieved pCR. Of the inclusion patients, 115 patients received adjuvant chemotherapy (Chemo group) and others did not receive therapy (No-chemo group) got close hospital follow-up care. The detailed comparison of concerning characteristics of Chemo group and No-chemo group was listed in Table 1. Of not, the pair-wise comparisons of all covariates were not significant with p more than 0.05 (Table 1), which demonstrated the characteristics were balanced between

Table 1 Clinical and demographics characteristics of patients with $\mathrm{pCR}$, stratified by adjuvant chemotherapy in the FUSCC

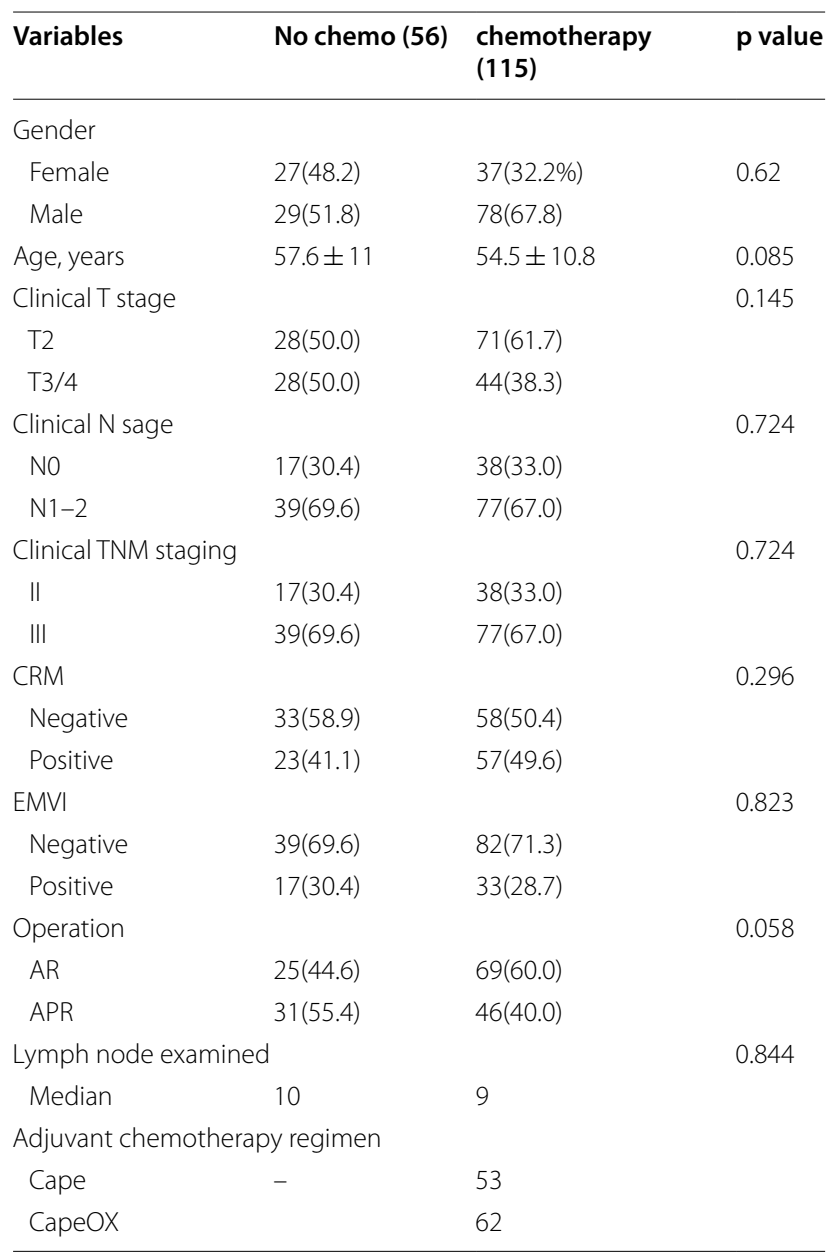

CRM, circumferential resection margin; EMVI, extramural venous invasion; $A R$, anterior resection; APR, abdominoperineal resection 
two treatments groups. Above all, the preoperational high-risk parameters also did not show any difference between the two groups, just like the circumferential resection margin(CRM), extramural venous invasion (EMVI).

\section{Overall survival}

Kaplan-Meier survival analysis did not reveal a significant difference in OS between those undergoing adjuvant chemotherapy or not. (log-rank test $=0.17$, $\mathrm{p}=0.676$ ) (Fig. 1a). And the per-protocol analysis demonstrated an HR of 0.737 for overall survivals $(95 \% \mathrm{CI}$ 0.176-3.093) for adjuvant chemotherapy. Multivariable Cox analysis also confirmed that adjuvant chemotherapy did not provide additional survival profit after achievement of pCR. In detail, among the patients receiving adjuvant chemotherapy, $94.6 \%$ were survived at the end of the follow-up period time, comparing to $94.5 \%$ of patients who did not receive chemo treatment. The 10-year cumulative overall survival was estimated comparable as $94.5 \%$ and $92.8 \%$ in those who received adjuvant chemotherapy or not. Additionally, the cumulative rectal overall survival was presented in the same pattern at $1,3,5$, and 10-year regarding to adjuvant chemotherapy (Table 4). In light of the varied chemotherapy regimens provided in the Chemo group, we further explored the outcomes among three patient cohorts: No chemo group, Cape group (MonoChemotherapy with Capecitabine) and Capeox group (combination chemotherapy with Capecitabine and Oxaliplatin). Consistently, not a significant difference was found in OS between the No chemo group and two chemotherapy regimens cohorts (Fig. 1b). Using conventional general logistic regression to test for interaction analyses, no interactions with any of the baseline characteristics were statistically significant with the adjuvant chemotherapy $(\mathrm{P}>0,05)$.

Then we stratified patients according to the clinical covariates, and identified the impact of adjuvant chemotherapy on survival in different subgroups. In first, based on the $\mathrm{T}$ stage, in both stage $\mathrm{T} 2$ patients and $\mathrm{T}$ stage $3 / 4$, the overall survival of adjuvant chemotherapy was comparable with that in the no chemo group, no matter Mono or Combined Chemotherapy was administered (Fig. 2a). The multivariable Cox model also did not show apparent OS benefits for chemotherapy in various T stage groups (HR: 0.939, 95\% CI 0.234-3.747), see Table 2. Above all, extramural venous invasion (EMVI) and circumferential resection margin (CRM) detected by high-resolution magnetic resonance imaging have been historically regarded as prognostic factors for rectal cancers, which significantly widened the opportunities to identify individual patient risk and adapted the treatment plan accordingly. In line with stage-subgroups, adjuvant chemotherapy did not have significant roles in subgroups of EMVI and CRM. Chemotherapy seemed not improve survival in patients with positive or negative EMVI and CRM (Fig. 2b, c). Furthermore, we performed Cox proportional model and got the same results: the HR offered by EMVI and CRM as (HR: 0.316; 95\% CI 0.039-2.568) and (HR: 3.539; 95\% CI 0.714-17.539) respectively, see Table 2.
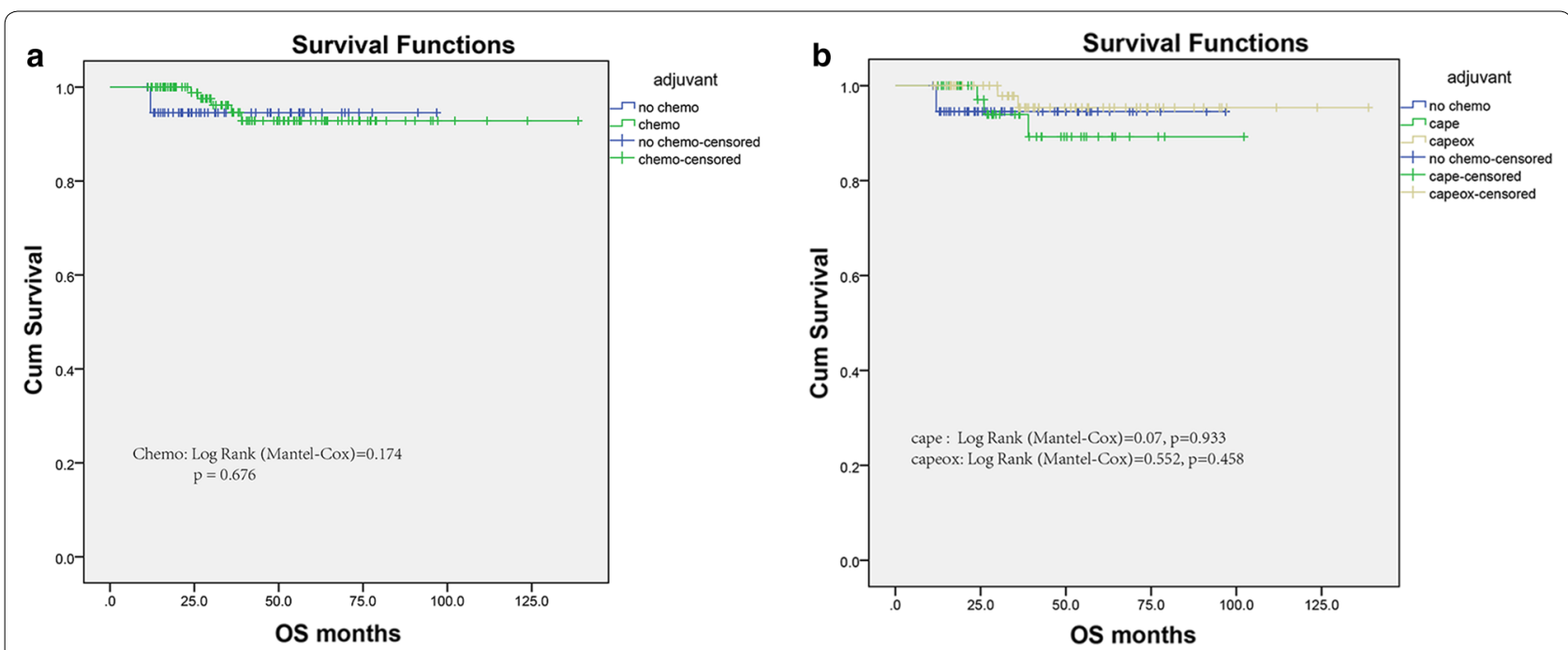

Fig. 1 Kaplan-Meier survival plots of overall survival (a) based on the receipt of adjuvant chemotherapy in the FUSCC. $\mathbf{b}$ stratified by the varied chemotherapy regimens provided in the Chemo group, Cape group (Mono-Chemotherapy with Capecitabine) and Capeox group (combination chemotherapy with Capecitabine and Oxaliplatin) 


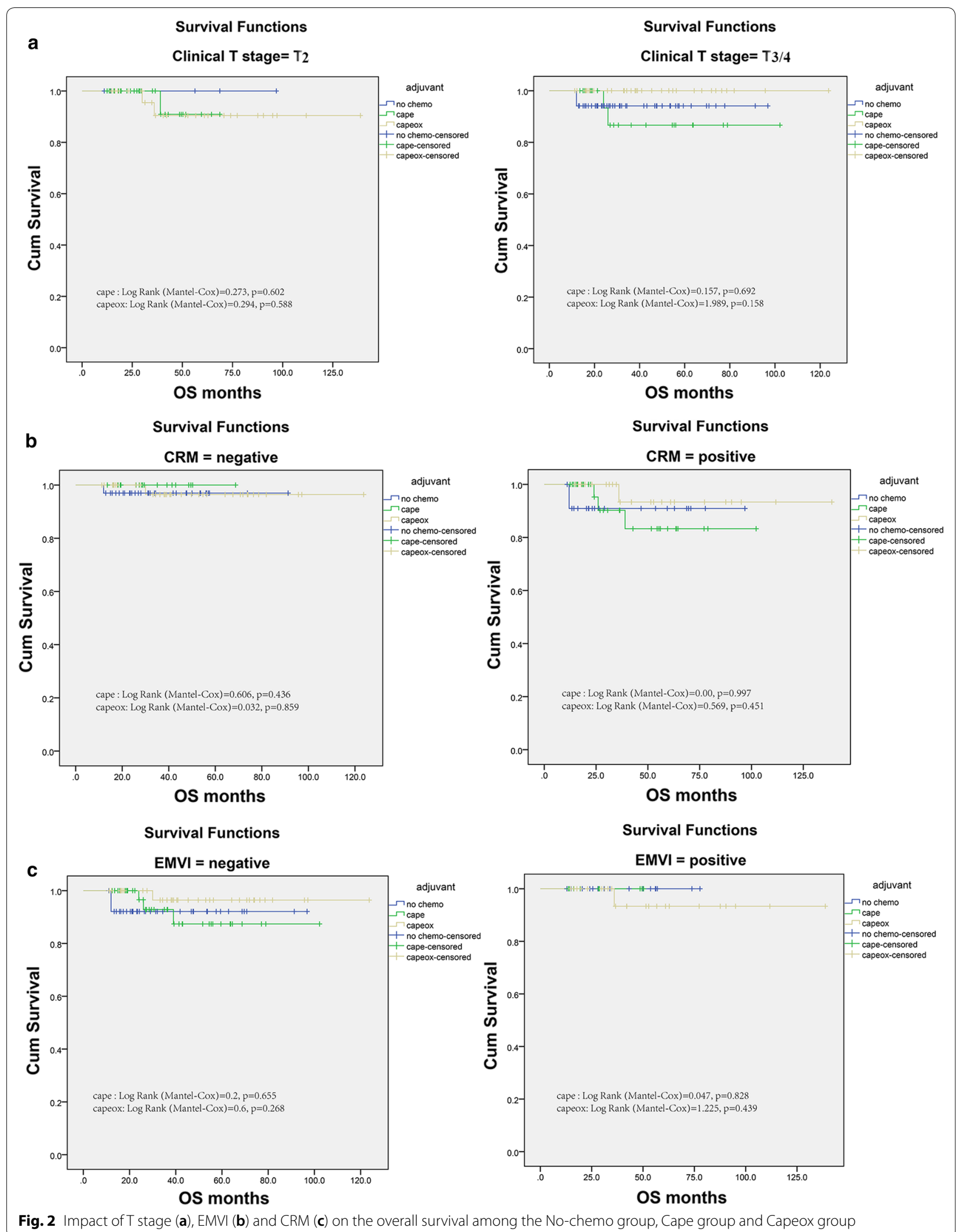


Table 2 Multivariate analyses of prognostic factors for overall survival in the FUSCC

\begin{tabular}{|c|c|c|c|}
\hline \multirow[t]{2}{*}{ Variables } & \multicolumn{3}{|c|}{ Cox proportional hazards } \\
\hline & Hazard ratio & $95 \% \mathrm{Cl}$ & p value \\
\hline Adjuvant chemotherapy & & & 0.678 \\
\hline No & 1 (reference) & & \\
\hline Yes & 0.739 & $0.176-3.093$ & \\
\hline Gender & & & 0.019 \\
\hline Female & 1 (reference) & & \\
\hline Male & 0.081 & $0.010-0.658$ & \\
\hline Age & 1.058 & $0.985-1.135$ & 0.12 \\
\hline Clinical T stage & & & 0.926 \\
\hline $\mathrm{T} 2$ & 1 (reference) & & \\
\hline $\mathrm{T} 3 / 4$ & 0.937 & $0.234-3.747$ & \\
\hline Clinical N sage & & & 0.697 \\
\hline No & 1 (reference) & & \\
\hline N1-2 & 0.753 & $0.180-3.150$ & \\
\hline CRM & & & 0.122 \\
\hline Negative & 1 (reference) & & \\
\hline Positive & 3.539 & $0.714-17.539$ & \\
\hline EMVI & & & 0.281 \\
\hline Negative & 1 (reference) & & \\
\hline Positive & 0.316 & $0.039-2.568$ & \\
\hline Operation & & & 0.361 \\
\hline$A R$ & 1 (reference) & & \\
\hline APR & 1.949 & $0.466-8.159$ & \\
\hline Lymph node examined & & & 0.064 \\
\hline$\leq 12$ & 1 (reference) & & \\
\hline$>12$ & 0.854 & $0.722-1.009$ & \\
\hline
\end{tabular}

CRM indicates circumferential resection margin; EMVI, extramural venous invasion; $A R$, anterior resection; $A P R$, abdominoperineal resection

\section{Disease-free survival}

Kaplan-Meier curves for disease-free survival were presented in Fig. 3a. The prognosis of Chemo group, with 5 -year disease-free survival of $87.5 \%$, was comparable with the No chemo group, with 5 -year disease-free survival of $88.8 \%(\mathrm{p}=0.854)$. what is more, Fig. 3b depicted the disease-free survival curves for colorectal patients with pCR estimated by 10 -year span of follow-up. Difference in survival were not found in patients with No chemo group, Mono and Combined Chemotherapy. And the per-protocol analysis demonstrated an HR of 0.912 for disease-free survival (95\% CI 0.342-2.432) for adjuvant chemotherapy. In addition, even combined regimen chemotherapy did not provide survival profit for $\mathrm{pCR}$ confirmed by multivariable Cox analysis, see Table 3 .

To identify the chemotherapy related prognostic factor linking DFS, Kaplan-Meier adjusted survival analysis stratified by $\mathrm{T}$ stage, EMVI and CRM were used to compare disease-free survival among No chemo group, Cape adjuvant chemotherapy and Capeox chemotherapy group. Similarly, there were no difference in disease-free survival with respect to positive for EMVI and CRM or not, no matter Mono or Combined Chemotherapy was administered (Fig. $4 \mathrm{a}-\mathrm{c}$ ). At the meantime, the results of the Cox proportional hazards analyses for factors associated with disease-free survival were summarized in Table 4. T stage (multivariate Cox HR, 0.697; 95\% CI 0.275-1.766; $\mathrm{p}=0.446$ ), EMVI (multivariate Cox HR, 0.462; 95\% CI 0.134-1.597; $\mathrm{p}=0.222$ ), and the status of CRM (multivariate Cox HR, 1.194; 95\% CI 0.474-3.010; $\mathrm{p}=0.707$ ) were not significantly associated with diseasefree survival.
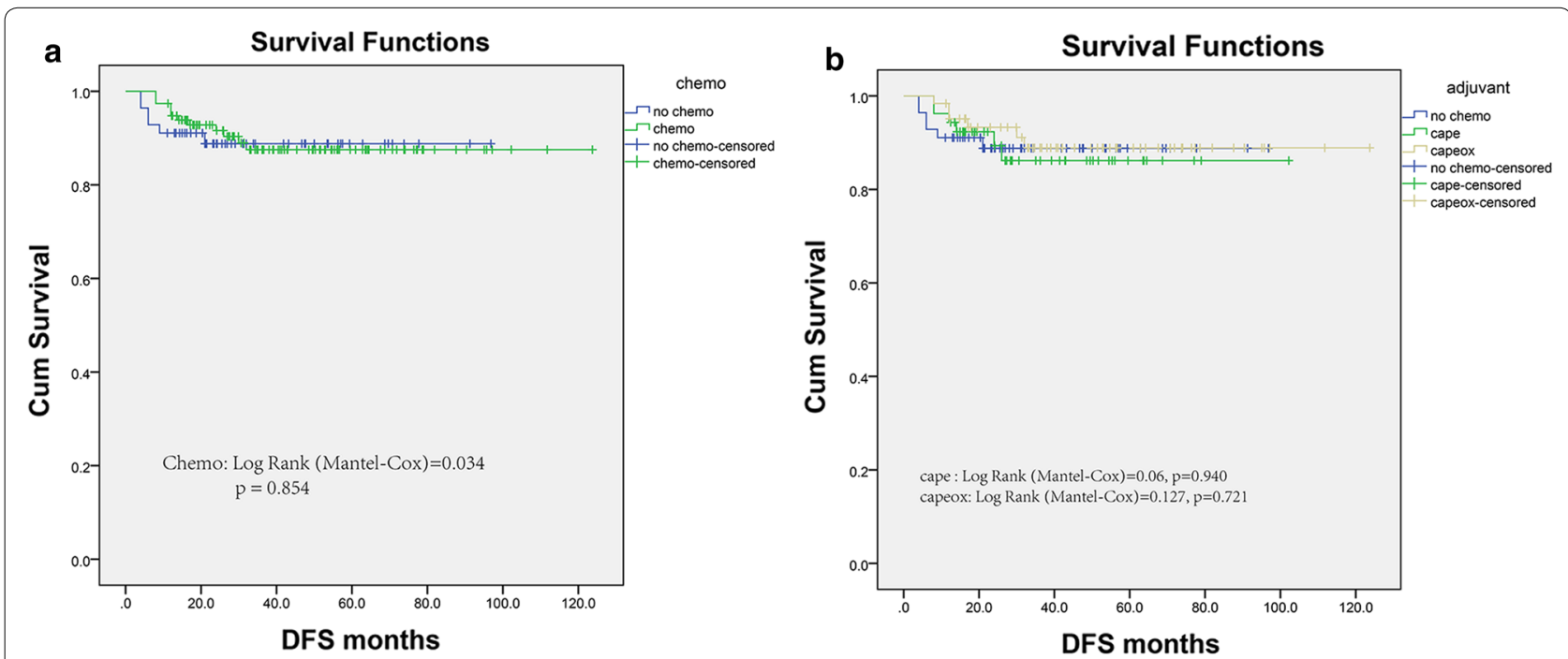

Fig. 3 Kaplan-Meier survival plots of disease-free survival (a) based on the receipt of adjuvant chemotherapy in the FUSCC. $\mathbf{b}$ stratified by the varied chemotherapy regimens provided in the Chemo group, Cape group and Capeox group 


\begin{tabular}{|c|c|c|c|}
\hline \multirow[t]{2}{*}{ Variables } & \multicolumn{3}{|c|}{ Cox proportional hazards } \\
\hline & Hazard ratio & $95 \% \mathrm{Cl}$ & $\mathrm{p}$ value \\
\hline Adjuvant chemotherapy & & & 0.855 \\
\hline No & 1 (reference) & & \\
\hline Yes & 0.912 & $0.342-2.432$ & \\
\hline Gender & & & 0.215 \\
\hline Female & 1 (reference) & & \\
\hline Male & 1.340 & $0.844-2.127$ & \\
\hline Age & 1.016 & $0.972-1.061$ & 0.481 \\
\hline Clinical T stage & & & 0.446 \\
\hline $\mathrm{T} 2$ & 1 (reference) & & \\
\hline $\mathrm{T} 3 / 4$ & 0.697 & $0.275-1.766$ & \\
\hline Clinical N sage & & & 0.401 \\
\hline No & 1 (reference) & & \\
\hline N1-2 & 0.657 & $0.247-1.751$ & \\
\hline CRM & & & 0.707 \\
\hline Negative & 1 (reference) & & \\
\hline Positive & 1.194 & $0.474-3.010$ & \\
\hline EMVI & & & 0.222 \\
\hline Negative & 1 (reference) & & \\
\hline Positive & 0.462 & $0.134-1.597$ & \\
\hline Operation & & & 0.391 \\
\hline$A R$ & 1 (reference) & & \\
\hline APR & 1.503 & $0.593-3.809$ & \\
\hline Lymph node examined & & & 0.381 \\
\hline$\leq 12$ & 1 (reference) & & \\
\hline$>12$ & 0.406 & $0.054-3.049$ & \\
\hline
\end{tabular}

CRM, circumferential resection margin; EMVI, extramural venous invasion; $A R$, anterior resection; APR, abdominoperineal resection

\section{Recurrences}

In total, there were 18 distant recurrences, with the most common sites being lung and liver, and no local recurrences occurred in the two groups. At 5 years, the cumulative incidence for distant recurrences was $12.5 \%$ in the chemotherapy group and $11.2 \%$ in the no chemotherapy group (HR 0.912, 95\% CI 0.342-2.432). Similar results were found in per-protocol analysis (HR 0.854, 95\% CI 0.71-1.36; $\mathrm{p}=0.41)$. Their cumulative incidence of relapse in terms of time interval post- surgery (Fig. 5a) revealed that the rate of relapse surged rapidly on the 12 months and rose up to peak $10.5 \%$ in the 36 th month. The percentage of relapse in the interval after surgery was $22.2,38.9$, and $16.6 \%$ in the 6 th, 12 th, and 18 th months, respectively (Fig. 5b).
Systematic comparison of adjuvant chemotherapy after achievement of pCR

In all, 96 related studies were identified from the PubMed and Embase search. After a one by one screening process for the titles and abstracts, 8 trials were selected for the final analysis. As a result, a total of 5491 patients with pCR was enrolled after a literature search performed in the electronic databases [8, 9, 13-18] (Table 5). Then we performed a systematic comparison of the available data to assess the efficacy of adjuvant chemotherapy for $\mathrm{pCR}$ treated with neoadjuvant treatment and radical surgery, using 5-year survival (OS and DFS) as endpoints.

Regarding to 5-year overall survival, the pooling data did not produce a statistically significant effect on survival prognosis in cases of adjuvant chemotherapy performed, $R R=0.79$, 95\% CI 0.5-1.25, $\mathrm{p}=0.311$, while heterogeneity was significant, $\mathrm{I}^{2}=77.5 \%$ (see Fig. $6 \mathrm{a}$ ). In addition, five-year DFS were also available for this comparison. Consistently, adjuvant chemotherapy similarly did not reduce the risk of disease progression ( $R R, 0.95$; 95\% CI 0.7-1.29; p =0.738; Fig. 6b). what is more, no statistical heterogeneity was detected, $\mathrm{I}^{2}=16.4 \%$.

To identify potential sources of heterogeneity, sensitivity evaluations were carried out and further to examine the influence of different inclusion standards on the overall survival estimate. A subgroup analysis suggested that adjuvant chemotherapy consistently did not affect the 5-year overall survival $(\mathrm{RR}=0.87,95 \% \mathrm{CI} 0.52-1.45, \mathrm{p}=0.586$, no heterogeneity observed, $\mathrm{I}^{2}=40.3 \%$, see Fig. $6 \mathrm{c}$ ), when baseline demographics characteristics balanced.

To test publication bias in the present study, visual inspection of Begg's funnel plot identified symmetry (Fig. 6d) and the Egger linear regression test and the Begg rank correlation test also revealed no evidence of bias $(\mathrm{p}=0.548)$.

\section{Discussion}

To our knowledge, this study is the first and largest study among Chinese population assessing the association between $\mathrm{pCR}$ and long-term prognosis. There are two main complementary findings highlighted in this study: 1) No association between improved OS and DFS and the reception of adjuvant chemotherapy after achievement of pCR; Meanwhile, the pretreatment factors of advanced T stage, positive EMVI and CRM remained no associated with worse outcomes in the context of a pCR. and 2) cumulative incidence of distant relapse surged rapidly on the 12 months and rose up to peak in the 36th month. 


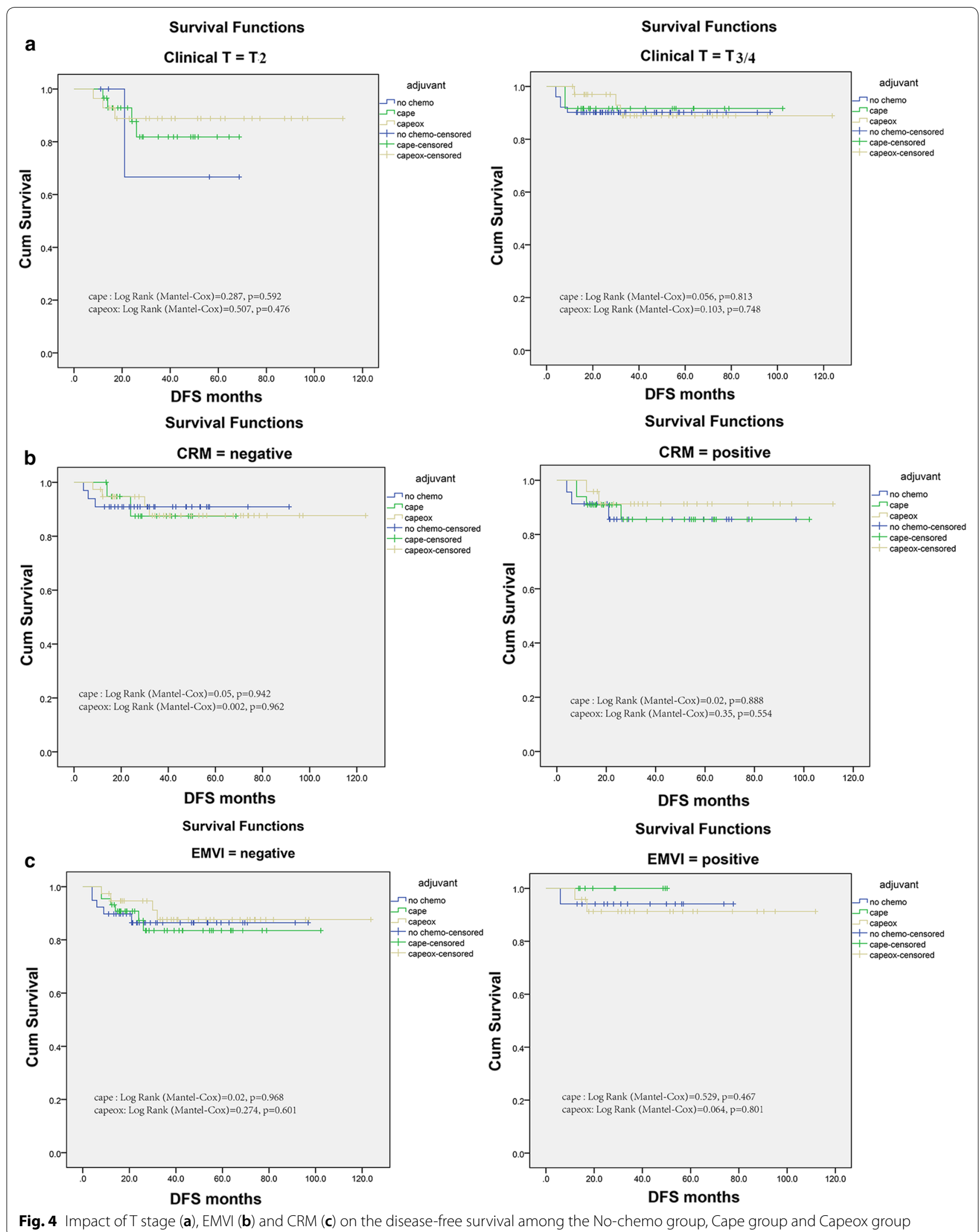


Table 4 Estimated year survival rates $(1,3,5$, and 10) in patients with $\mathrm{PCR}$, stratified by adjuvant chemotherapy in the FUSCC

\begin{tabular}{lcl}
\hline Survival & No chemo & Chemotherapy \\
\hline Overall survival (patients at risk) & & \\
1-year rate & $94.5 \pm 3.1(56)$ & $98.8 \pm 1.2(115)$ \\
3-year rate & $94.5 \pm 3.1(22)$ & $94.6 \pm 1.2(53)$ \\
5-year rate & $94.5 \pm 3.1(7)$ & $92.8 \pm 3.1(22)$ \\
10-year rate & $94.5 \pm 3.1(1)$ & $92.8 \pm 3.1(1)$ \\
Disease-free survival (patients at risk) & \\
1-year rate & $91.1 \pm 3.8(56)$ & $93.8 \pm 2.3(115)$ \\
3-year rate & $88.8 \pm 4.3(20)$ & $92.8 \pm 2.5(49)$ \\
5-year rate & $88.8 \pm 4.3(6)$ & $87.5 \pm 3.5(21)$ \\
10-year rate & $88.8 \pm 4.3(0)$ & $87.5 \pm 3.5(0)$ \\
\hline
\end{tabular}

This current study is unique in comparing adjuvant chemotherapy against observation alone in context of pCR. In fact, previously there also existed some small sample size trials or sub-group analysis concerning to the role of adjuvant chemotherapy for patients with pCR. Consistently, those who received adjuvant chemotherapy had no survival benefits compared to those without adjuvant chemotherapy on rectal cancers with pCR [8]. Similarly, even during a median follow-up of 57 months, the 5y-DFS rates for patients in the chemo group and the no-chemo group were also comparable, showing no significant difference [9]. However, the retrospective design nature, small size sample and selection bias of those studies frequently were considered to be the potential drawbacks, hence, no consensus was arrived on the basis of pCR in determining the necessity for adjuvant chemotherapy.

Here a largest baseline balanced cohort demonstrated adjuvant chemotherapy had no overall and disease-free survival profit in $\mathrm{PCR}$, which added more clear evidence on the no benefit of chemotherapy applied in pCR rectal cancers. The main reason was lied to accurate estimation for post-operative pathology, as final pathologic stage is most prognostic of oncologic outcomes in locally advanced rectal cancer patients after neo-adjuvant chemoradiotherapy [19]. It can be determined that most predictive of overall and disease-free survival was based on final pathologic $\mathrm{T}$ classification and $\mathrm{N}$ classification elements, while this applicability to the present pCR population in question is direct. Moreover, besides tumor response to preoperative therapy and final pathologic stage as strong predictors of survival, oncological outcome is most influenced by preoperative $\mathrm{T}$ stage and high-risk factors, such as EMVI and CRM [20, 21]. Then we stratified patients according to the clinical covariates, and identified the impact of adjuvant chemotherapy on survival in different subgroups. Similarly, there were no differences in survival with respect to positive for EMVI and CRM or not, no matter Mono or Combined Chemotherapy was administered. In addition, chemotherapy may be more effective in patients with poor prognosis, such as those with stage III or stage II with T3-4, just as the ADORE trial examining the role of oxaliplatin and leucovorin as chemotherapy for rectal cancer, it turned out that patients with final pathological stage III received improving survival but patients with stage I and II disease did not [22]. That is why we did not observe any survival profit in $\mathrm{pCR}$ when adding chemotherapy. Additionally, achievement of pCR seemed to have an excellent prognosis regardless of utilization of adjuvant chemotherapy, or the potential benefit of chemotherapy in patients with a pCR after neo-chemoradiation would appear to be small if it exists at all. The only way to prove the absence of such a small benefit must require a large sample size trial. That is why our study has been carried out here, as this pooled analysis covered not only 5491 patients with $\mathrm{pCR}$ in the
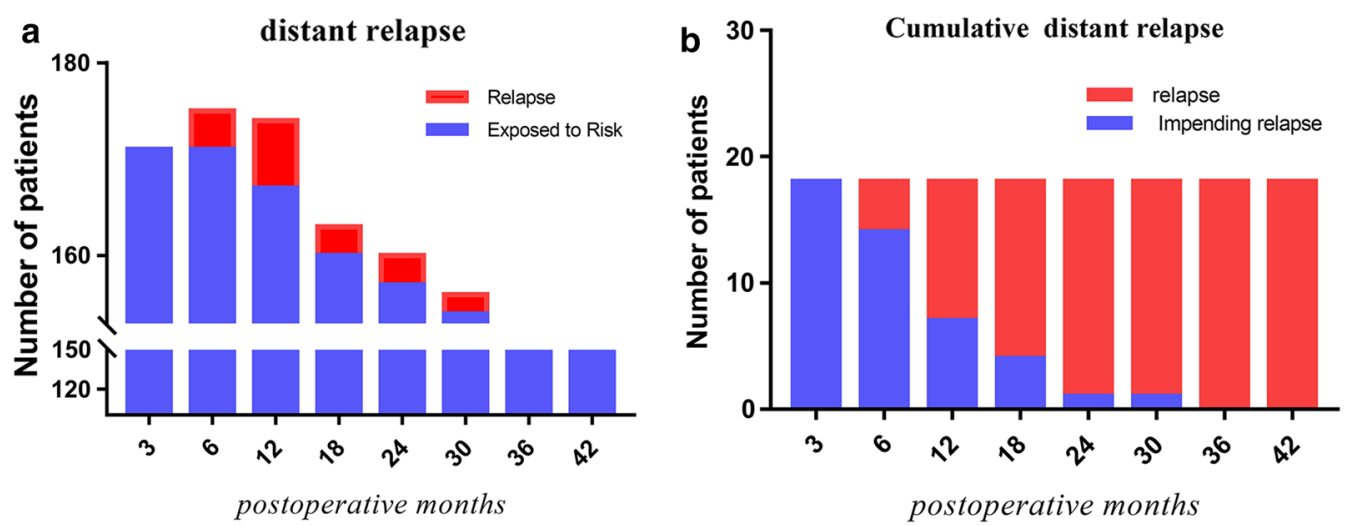

Fig. 5 a Number of distant relapse in pCR in relation to time at the end of radical surgery. $\mathbf{b}$ Cumulative numbers of distant relapse relative to the all relapse with the post-operation months 
Table 5 Overview of baseline characteristics per included study

\begin{tabular}{|c|c|c|c|c|c|c|c|c|c|}
\hline Author & Shahab D & Zhou & Geva R & $\begin{array}{l}\text { Gamaleldin } \\
M\end{array}$ & Kuan FC & Kiran RP & HuX & CAPIRCI C & Dossa F \\
\hline Year & 2017 & 2016 & 2014 & 2017 & 2017 & 2012 & 2019 & 2008 & 2018 \\
\hline Country & USA & China & Israel & USA & China & USA & China & Italy & Canada \\
\hline Study design & Retrospective & Case control & Case control & $\begin{array}{c}\text { Prospective } \\
\text { control }\end{array}$ & Case control & Case control & Case control & $\begin{array}{l}\text { Prospective } \\
\text { sub-group }\end{array}$ & Retrospective \\
\hline $\mathrm{N}$ & $789 / 2102$ & $19 / 21$ & $35 / 17$ & $47 / 83$ & $114 / 145$ & $14 / 34$ & $115 / 56$ & $127 / 439$ & $667 / 667$ \\
\hline $\operatorname{Age}(c / n)$ & $57.2 / 61.2$ & $54 / 54.1$ & $60.9 / 68.7$ & $55.9 / 60.6$ & $56.7 / 61.8$ & $50.5 / 55.5$ & $54.5 / 57.6$ & 62 & $56 / 57$ \\
\hline $\begin{array}{l}\text { Gender } \\
\text { (male\%) }\end{array}$ & $56.8 / 61.2$ & $36.8 / 76.2$ & $68.5 / 47$ & $57.4 / 67.5$ & $59.6 / 66.2$ & $50 / 73.5$ & $67.8 / 57.8$ & 67 & $56.8 / 55.5$ \\
\hline \multicolumn{10}{|l|}{ cT-stage } \\
\hline $\mathrm{T} 1-2$ & $110 / 348$ & $2 / 2$ & $1 / 2$ & NA & $11 / 21$ & $1 / 4$ & $57 / 23$ & 29 & $60 / 59$ \\
\hline T3-4 & $679 / 1754$ & $17 / 19$ & $31 / 14$ & NA & $102 / 124$ & $13 / 30$ & $58 / 33$ & 409 & $585 / 584$ \\
\hline \multicolumn{10}{|l|}{ cN-stage } \\
\hline No & $388 / 1224$ & $6 / 7$ & $23 / 12$ & $25 / 48$ & $33 / 52$ & $9 / 22$ & $66 / 31$ & NA & $319 / 319$ \\
\hline $\mathrm{N}+$ & $401 / 878$ & $10 / 12$ & $9 / 3$ & $22 / 34$ & $81 / 91$ & $5 / 12$ & $49 / 25$ & 169 & $348 / 348$ \\
\hline \multicolumn{10}{|l|}{ Surgery } \\
\hline$A R$ & $565 / 1474$ & $16 / 12$ & $26 / 10$ & $5 / 41$ & $78 / 108$ & $12 / 22$ & $69 / 25$ & 339 & NA \\
\hline APR & $183 / 511$ & $3 / 9$ & $9 / 7$ & $42 / 13$ & $15 / 16$ & $2 / 12$ & $46 / 31$ & 100 & NA \\
\hline $\begin{array}{l}\text { Chemo- } \\
\text { therapy } \\
\text { regimen }\end{array}$ & NA & $\begin{array}{l}\text { Combined } \\
\text { and single- } \\
\text { agent }\end{array}$ & NA & NA & NA & NA & $\begin{array}{l}\text { Combined } \\
\text { and single- } \\
\text { agent }\end{array}$ & $\begin{array}{l}\text { Combined } \\
\text { and single- } \\
\text { agent }\end{array}$ & NA \\
\hline
\end{tabular}

c/n, chemotherapy group vs. no-chemo group; NA, no available; AR, anterior resection; APR, abdominoperineal resection

whole world, but also the carefully followed patients at our single center. In total, the sample size more than 4000 pCR was enough to reach a power of consolidated conclusion, while, the benefit of adjuvant chemotherapy cannot be detected on the patients with pCR.

However, against our study, a potential advantage providing from adjuvant chemotherapy was demonstrated in three retrospective studies [14, 18, 23]. In fact, those studies were all based on the National Cancer Database (NCDB). Although the patients sample seemed very large, while, the surgical procedure was not clarified sufficiently, just demonstrating Partial proctectomy or Total proctectomy. However, others studies including our FUSCC data, which did not observe benefit of adjuvant chemotherapy in pCR, all performed TME. That may be the major reason accounting for the discrepancy. In another word, those patients from NCDB with pCR might undergo local resection, instead of TME, devoid of reginal lymph nodes resection. In addition, another explanation can be attributed to the fact that report only presented overall survival rather than DFS in their analyses, which allowed not cancer-related comorbidities to affect survival outcomes. Given that the tumor behavior is routinely evaluated by DFS, DFS is one of the most sensitive factors of the intended effects of biological characteristic. Unlike overall survival, DFS is less influenced by disparities in the treatment of relapse disease, management of comorbidity, and differential rates of death from competing causes irrelative to cancer. Additionally, the retrospective design and unbalanced baseline of that study may be considered to be other additional potential reasons.

Generally, approximately $80 \%$ of disease recurrences after radical surgery of colorectal cancer occurred within the first 2 years post-surgery [24, 25]. Nevertheless, it does not appear to be the case for achievement of pCR in our study. It inferred from some studies that about $80 \%$ of the recurrences occur within the first 4 years, which was postponed in patients with pCR [26]. That parallels the findings here, as among the cases developing distant disease relapse, relapse remained occur more than 3 years after the surgery. We therefore recommended the perspective that achievement of $\mathrm{pCR}$ require more prolonged close follow care [27].

Owing to insufficient data, we were unable to perform a subanalysis for different basic characters or concomitances, such as the lymph nodes retrieved and clinical TNM staging, which had important implication in the fates of outcome. Elsely, reporting bias is inherent in any retrospective database, so appropriate adjustment for potential confounders is performed to validate the effect of adjuvant chemotherapy. Although no heterogeneity in the pooling analysis was found in the present 


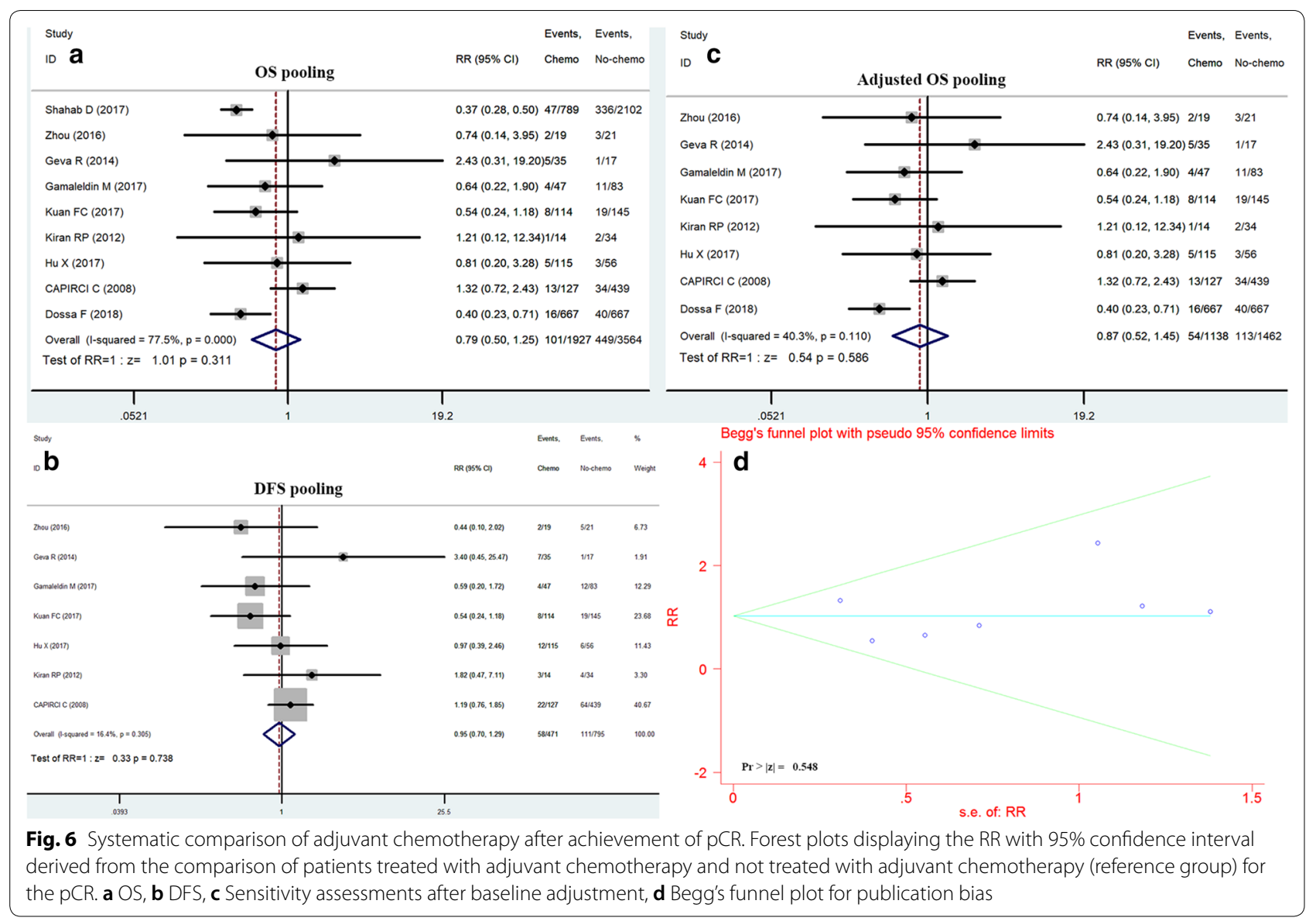

analysis, the results of a large-scale, dedicated, randomized controlled trials are awaited to determine the external validity of our findings.

\section{Conclusion}

Rectal cancer patients with pCR did not benefit from adjuvant chemotherapy after preoperative chemoradiotherapy and radical surgery, which supports the results of previous studies investigating the role of adjuvant chemotherapy in yield pathological stage. The value of adjuvant chemotherapy for pCR patients should be further determined in prospective randomized trials or large multicenter cohort studies, in addition, we recommended that achievement of $\mathrm{pCR}$ require more prolonged close follow care in case of distant metastasis.

\section{Abbreviations}

CRT: neo-adjuvant chemoradiotherapy; CRC: colorectal cancer; pCR: pathology completed response; FUSCC: Fudan University Shanghai Cancer Center; OS: overall survival; DFS: disease-free survival; CRM: circumferential resection margin; EMVI: extramural venous invasion.

\section{Acknowledgements}

Not applicable.

\section{Authors' contributions}

Conceived and designed the experiments: HX, PJJ and CSJ. Analysed the data: HX, LYQ, CSJ, MXJ. Contributed reagents/materials/analysis tools: HX and ZL. Wrote the paper: HX. All authors read and approved the final manuscript.

\section{Funding}

None.

\section{Availability of data and materials}

All the data used to support the findings of this study are included within the article. Please contact author for data requests.

\section{Ethics approval and consent to participate}

Not applicable.

\section{Consent for publication}

Not applicable.

\section{Competing interests}

The authors declare that they have no competing interests.

\section{Author details}

${ }^{1}$ Department of Colorectal Surgery, Fudan University Shanghai Cancer Center, 270 Dong'an Road, Shanghai 20032, China. ${ }^{2}$ Department of Oncology, Shanghai Medical College, Fudan University, Shanghai 200032, China.

Received: 19 February 2019 Accepted: 7 May 2019

Published online: 14 May 2019 


\section{References}

1. Scott NA, Susnerwala S, Gollins S, Myint AS, Levine E. Preoperative neoadjuvant therapy for curable rectal cancer-reaching a consensus 2008 . Colorectal Dis. 2009;11(3):245-8.

2. Sauer R, Fietkau R, Wittekind C, Martus P, Rödel C, Hohenberger W, et al. Adjuvant versus neoadjuvant radiochemotherapy for locally advanced rectal cancer. A progress report of a phase-III randomized trial (protocol CAO/ARO/AIO-94). Strahlenther Onkol. 2001;177(4):173-81.

3. Quasar Collaborative G, Gray R, Barnwell J, McConkey C, Hills RK, Williams NS, et al. Adjuvant chemotherapy versus observation in patients with colorectal cancer: a randomised study. Lancet. 2007;370(9604):2020-9.

4. Maas M, Nelemans PJ, Valentini V, Das P, Rodel C, Kuo LJ, et al. Long-term outcome in patients with a pathological complete response after chemoradiation for rectal cancer: a pooled analysis of individual patient data. Lancet Oncol. 2010;11(9):835-44.

5. Benson AB, Venook AP, Bekaii-Saab T, Chan E, Chen YJ, Cooper HS, et al. Rectal Cancer, Version 2. 2015. J Natl Compr Cancer Netw. 2015:13(6):719-28 (quiz 28)

6. Glynne-Jones R, Wyrwicz L, Tiret E, Brown G, Rödel C, Cervantes A, et al. Rectal cancer: ESMO Clinical Practice Guidelines for diagnosis, treatment and follow-up. Ann Oncol. 2017;28(suppl_4):iv22-40.

7. Hong TS, Ryan DP. Adjuvant chemotherapy for locally advanced rectal cancer: is it a given? J Clin Oncol. 2015;33(17):1878-80.

8. Kuan FC, Lai CH, Ku HY, Wu CF, Hsieh MC, Liu TW, et al. The survival impact of delayed surgery and adjuvant chemotherapy on stage II/III rectal cancer with pathological complete response after neoadjuvant chemoradiation. Int J Cancer. 2017:140(7):1662-9.

9. Zhou J, Qiu H, Lin G, Xiao Y, Wu B, Wu W, et al. Is adjuvant chemotherapy necessary for patients with pathological complete response after neoadjuvant chemoradiotherapy and radical surgery in locally advanced rectal cancer? Long-term analysis of $40 \mathrm{ypCR}$ patients at a single center. Int J Colorectal Dis. 2016;31(6):1163-8.

10. Chen WT, Ke TW, Li CC, Chien CR. Questionable role of adjuvant chemotherapy in rectal cancer patients who had reached pathological complete response after neoadjuvant concurrent chemoradiotherapy: no matter in the East or in the West. J Cancer Res Clin Oncol. 2014;140(9):1495-6.

11. Nitsche U, Maak M, Schuster T, Kunzli B, Langer R, Slotta-Huspenina J, et al. Prediction of prognosis is not improved by the seventh and latest edition of the TNM classification for colorectal cancer in a single-center collective. Ann Surg. 2011;254(5):793-800 (discussion-1)

12. Clarke MO. Cochrane reviewer's handbook 4.1.3[updated June 2001]. In: The Cochrane Library, Issue 3: Oxford: Update Software. 2001.

13. Geva R, Itzkovich E, Shamai S, Shacham-Shmueli E, Soyfer V, Klausner JM, et al. Is there a role for adjuvant chemotherapy in pathological complete response rectal cancer tumors following neoadjuvant chemoradiotherapy? J Cancer Res Clin Oncol. 2014;140(9):1489-94.

14. Shahab D, Gabriel E, Attwood K, Ma WW, Francescutti V, Nurkin S, et al. Adjuvant chemotherapy is associated with improved overall survival in locally advanced rectal cancer after achievement of a pathologic complete response to chemoradiation. Clin Colorectal Cancer. 2017;16(4):300-7.

15. Gamaleldin M, Church JM, Stocchi L, Kalady M, Liska D, Gorgun E. Is routine use of adjuvant chemotherapy for rectal cancer with complete pathological response justified? Am J Surg. 2017;213(3):478-83.
16. Kiran RP, Kirat HT, Burgess AN, Nisar PJ, Kalady MF, Lavery IC. Is adjuvant chemotherapy really needed after curative surgery for rectal cancer patients who are node-negative after neoadjuvant chemoradiotherapy? Ann Surg Oncol. 2012;19(4):1206-12.

17. Capirci C, Valentini V, Cionini L, De Paoli A, Rodel C, Glynne-Jones R, et al. Prognostic value of pathologic complete response after neoadjuvant therapy in locally advanced rectal cancer: long-term analysis of 566 ypCR patients. Int J Radiat Oncol Biol Phys. 2008;72(1):99-107.

18. Dossa F, Acuna SA, Rickles AS, Berho M, Wexner SD, Quereshy FA, et al. Association between adjuvant chemotherapy and overall survival in patients with rectal cancer and pathological complete response after neoadjuvant chemotherapy and resection. JAMA Oncol. 2018;4(7):930-7.

19. Quah HM, Chou JF, Gonen M, Shia J, Schrag D, Saltz LB, et al. Pathologic stage is most prognostic of disease-free survival in locally advanced rectal cancer patients after preoperative chemoradiation. Cancer. 2008:113(1):57-64.

20. Siddiqui MRS, Simillis C, Hunter C, Chand M, Bhoday J, Garant A, et al. A meta-analysis comparing the risk of metastases in patients with rectal cancer and MRI-detected extramural vascular invasion (mrEMVI) vs mrEMVI-negative cases. Br J Cancer. 2017;116(12):1513-9.

21. Sung S, Kim SH, Lee JH, Nam TK, Jeong S, Jang HS, et al. Continuous effect of radial resection margin on recurrence and survival in rectal cancer patients who receive preoperative chemoradiation and curative surgery: a multicenter retrospective analysis. Int J Radiat Oncol Biol Phys. 2017;98(3):647-53.

22. Hong YS, Nam BH, Kim KP, Kim JE, Park SJ, Park YS, et al. Oxaliplatin, fluorouracil, and leucovorin versus fluorouracil and leucovorin as adjuvant chemotherapy for locally advanced rectal cancer after preoperative chemoradiotherapy (ADORE): an open-label, multicentre, phase 2, randomised controlled trial. Lancet Oncol. 2014;15(11):1245-53.

23. Turner MC, Keenan JE, Rushing CN, Gulack BC, Nussbaum DP, Benrashid E, et al. Adjuvant chemotherapy improves survival following resection of locally advanced rectal cancer with pathologic complete response. J Gastrointest Surg. 2019. https://doi.org/10.1007/s1 1605-018-04079-8.

24. Scholefield JH, Steele RJ, British Society For G, Association of Coloproctology for Great B, Ireland. Guidelines for follow up after resection of colorectal cancer. Gut. 2002;51(Suppl 5):V3-5.

25. Umpleby HC, Fermor B, Symes MO, Williamson RC. Viability of exfoliated colorectal carcinoma cells. Br J Surg. 1984;71(9):659-63.

26. Valentini V, van Stiphout RG, Lammering G, Gambacorta MA, Barba MC, Bebenek M, et al. Selection of appropriate end-points ( $p C R$ vs 2yDFS) for tailoring treatments with prediction models in locally advanced rectal cancer. Radiother Oncol. 2015;114(3):302-9.

27. Habr-Gama A, Perez RO, Proscurshim I, Campos FG, Nadalin W, Kiss D, et al. Patterns of failure and survival for nonoperative treatment of stage c0 distal rectal cancer following neoadjuvant chemoradiation therapy. J Gastrointest Surg. 2006;10(10):1319-28 (discussion 28-9).

\section{Publisher's Note}

Springer Nature remains neutral with regard to jurisdictional claims in published maps and institutional affiliations.

Ready to submit your research? Choose BMC and benefit from

- fast, convenient online submission

- thorough peer review by experienced researchers in your field

- rapid publication on acceptance

- support for research data, including large and complex data types

- gold Open Access which fosters wider collaboration and increased citations

- maximum visibility for your research: over 100M website views per year

At $\mathrm{BMC}$, research is always in progress.

Learn more biomedcentral.com/submissions 